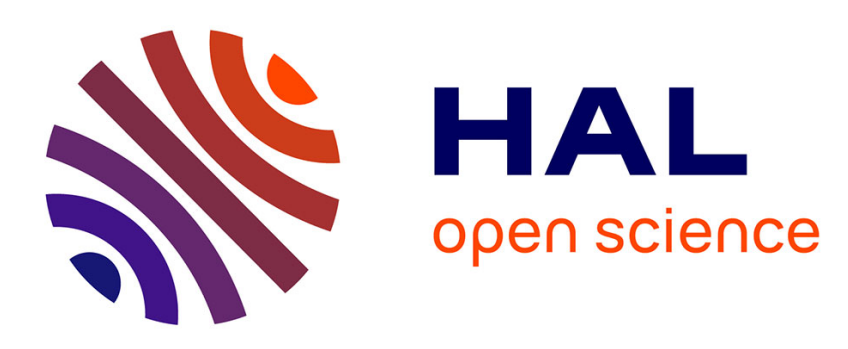

\title{
Review of Shape Deviation Modeling for Additive Manufacturing
}

\author{
Zuowei Zhu, Safa Keimasi, Nabil Anwer, Luc Mathieu, Lihong Qiao
}

\section{To cite this version:}

Zuowei Zhu, Safa Keimasi, Nabil Anwer, Luc Mathieu, Lihong Qiao. Review of Shape Deviation Modeling for Additive Manufacturing. International Joint Conference on Mechanics, Design Engineering \& Advanced Manufacturing (JCM 2016), Sep 2016, Catane, Italy. pp.241-205, 10.1007/978-3-31945781-9_25. hal-01363769

\section{HAL Id: hal-01363769 https://hal.science/hal-01363769}

Submitted on 11 Sep 2016

HAL is a multi-disciplinary open access archive for the deposit and dissemination of scientific research documents, whether they are published or not. The documents may come from teaching and research institutions in France or abroad, or from public or private research centers.
L'archive ouverte pluridisciplinaire HAL, est destinée au dépôt et à la diffusion de documents scientifiques de niveau recherche, publiés ou non, émanant des établissements d'enseignement et de recherche français ou étrangers, des laboratoires publics ou privés. 


\title{
Review of Shape Deviation Modeling for Additive Manufacturing
}

\author{
Zuowei ZHU $^{1}$, Safa KEIMASI ${ }^{1}$, Nabil ANWER ${ }^{1 *}$, Luc MATHIEU ${ }^{1}$ and \\ Lihong QIAO ${ }^{2}$
}

${ }^{1}$ LURPA, ENS Cachan, Univ. Paris-Sud, Université Paris-Saclay, 94235 Cachan, France

${ }^{2}$ School of Mechanical Engineering and Automation, Beihang University, Beijing 100191, China

*Corresponding author. Tel.: +33-(0)147402413; fax: +33-(0)147402220. E-mail address: anwer@lurpa.ens-cachan.fr

\begin{abstract}
Additive Manufacturing (AM) is becoming a promising technology capable of building complex customized parts with internal geometries and graded material by stacking up thin individual layers. However, a comprehensive geometric model for Additive Manufacturing is not mature yet. Dimensional and form accuracy and surface finish are still a bottleneck for AM regarding quality control. In this paper, an up-to-date review is drawn on methods and approaches that have been developed to model and predict shape deviations in AM and to improve geometric quality of AM processes. A number of concluding remarks are made and the Skin Model Shapes Paradigm is introduced to be a promising framework for integration of shape deviations in product development, and the digital thread in AM.
\end{abstract}

Keywords: Additive manufacturing, Geometric deviation, Skin model shapes, Geometric modeling.

\section{Introduction}

Additive Manufacturing (AM), as a most frequently used method for rapid prototyping nowadays, was first explored and applied in the automotive, aerospace and medical industries and it is considered to be one of the pillars of the fourth industrial revolution. Different from traditional machining, in which parts are made by removing materials from a larger stock through different processes, AM fabricates volumes layer by layer from their three-dimensional CAD model data.

Additive Manufacturing and its different technologies have been reviewed comprehensively by many authors. A classification of additive manufacturing technologies is shown in Table 1 according to their characteristics. 
Table 1. Process categories of additive manufacturing as classified by ASTM [1].

\begin{tabular}{|c|c|c|}
\hline Process type & Description & Related Technologies \\
\hline Binder jetting & $\begin{array}{l}\text { Liquid bonding agent selective- } \\
\text { ly deposited to join powder }\end{array}$ & $\begin{array}{l}\text { Powder bed and inkjet head (PBIH), } \\
\text { plaster-based 3D printing (PP) }\end{array}$ \\
\hline Material jetting & $\begin{array}{l}\text { Droplets of build material se- } \\
\text { lectively deposited }\end{array}$ & Multi-jet modeling (MJM) \\
\hline $\begin{array}{l}\text { Powder bed fu- } \\
\text { sion }\end{array}$ & $\begin{array}{l}\text { Thermal energy selectively fus- } \\
\text { es regions of powder bed }\end{array}$ & $\begin{array}{l}\text { Electron beam melting (EBM), selective } \\
\text { laser sintering (SLS) }\end{array}$ \\
\hline $\begin{array}{l}\text { Directed energy } \\
\text { deposition }\end{array}$ & $\begin{array}{l}\text { Focused thermal energy melts } \\
\text { materials as deposited }\end{array}$ & Laser metal deposition (LMD) \\
\hline Sheet lamination & $\begin{array}{l}\text { Sheets of material bonded } \\
\text { together }\end{array}$ & $\begin{array}{l}\text { Laminated object manufacturing (LOM), } \\
\text { ultrasonic consolidation(UC) }\end{array}$ \\
\hline $\begin{array}{l}\text { Vat photopoly- } \\
\text { merization }\end{array}$ & $\begin{array}{l}\text { Liquid photopolymer selective- } \\
\text { ly cured by light activation }\end{array}$ & $\begin{array}{l}\text { Stereolithography (SLA), digital light } \\
\text { processing (DLP) }\end{array}$ \\
\hline $\begin{array}{l}\text { Material extru- } \\
\text { sion }\end{array}$ & $\begin{array}{l}\text { Material selectively dispensed } \\
\text { through nozzle or orifice }\end{array}$ & Fused deposition modeling (FDM) \\
\hline
\end{tabular}

A brief illustration of the digital chain of an AM process is shown in Figure 1. The process can be divided into an input phase, a build phase and an output phase. In the input phase, the CAD model of the part is designed and converted as a STereoLithography (STL) file format that is readable for AM machines and provides the geometry information. This STL file is basically an approximation of the designed part achieved by triangulation, which causes deviation in the final part. In the build phase, process parameters like energy source, layer thickness, build direction, supports and material constraints are set in the machine and the part is fabricated layer by layer. The output phase is an imperative part of the process, in which procedures like support removal, cleaning, heat treatment and NC machining are executed to ensure the final quality of the part.

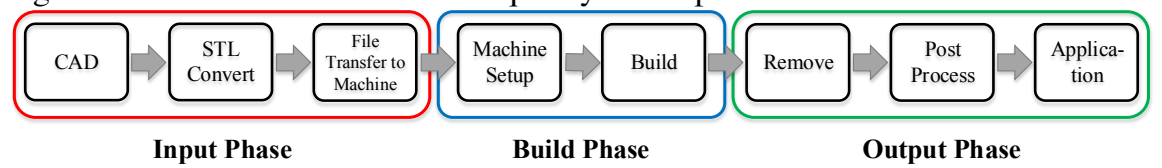

Fig. 1. Typical digital chain of an AM process.

Factors arising from each phase may introduce geometric deviations of the final part, including quality of input file, machine errors, build orientation, process parameters, material shrinkage and staircase effects due to layer thickness. The control of geometrical accuracy remains a major bottleneck in the application of AM. The Skin Model Shapes (SMS) paradigm, which stems from the theoretical foundations of Geometrical Product Specification and Verification, is proposed as a comprehensive framework to capture product shape variability in different phases of product lifecycle [2]. It enables the consideration of geometric deviations that are expected, predicted or already observed in real manufacturing processes. In- 
depth researches into the integration of thermal effects in its application on tolerance analysis have paved the way for its adaptation to deviation modeling in the context of additive manufacturing [3].

In this paper, a review will be done on the methods developed to model and predict shape deviations in AM and to improve geometric quality of AM processes. Some concluding remarks will be made and the Skin Model Shapes paradigm will be introduced to be a promising framework for modeling shape deviations in AM.

\section{Review of shape deviation modeling for AM}

In order to model the shape deviations and improve the geometrical accuracy in $\mathrm{AM}$ processes, in recent years, numbers of researchers have proposed different models and approaches regarding to specific error sources as well as AM processes. Since the AM process is almost automatic, most of these studies have been devoted to improving the design, among which two major categories of approaches can be distinguished. One category focuses on changing the CAD design by compensating the shape deviations based on predictive deviation models. The other category focuses on modifying the input files or improving the slicing techniques of AM processes.

\subsection{Compensation of shape deviations}

The researches with respect to deviation compensation are conducted for different purposes. The compensation models can be briefly classified into machine error compensation and part shrinkage compensation.

Tong et al. [4,5] proposes a parametric error model, which models all the repeatable errors of SLA (Stereolithography) machine and FDM (Fused Deposition Modeling) machine with generic parametric error functions. The coefficients of the functions can be estimated through regression of the measurement data gathered from given points. The model can then be used to analyze individual deviations in the Cartesian Coordinate System (CCS), based on which appropriate compensations can be made on the input files to minimize the shape deviations of the final products. However, since the deviations are estimated from individual points, the continuity of product geometry is not considered and the accuracy of estimation depends largely on the selected AM machine and measurement points, which lacks generality.

A series of studies conducted by Huang $[6,7,8,9,10]$ and his research team have been dedicated to developing a predictive model of geometric deviations that is able to learn from the deviation information obtained from a certain number of tested product shapes and derive compensation plans for new and untested prod- 
ucts. They aim at establishing a generic methodology that is independent of shape complexity and specific AM processes. Their first attempt [6,7] initiates in modeling the in-plane shape deviations induced by specific influential factors in the MIP-SLA and FDM processes, based on which they develop a generic approach to model and predict in-plane deviation caused by shape shrinkages along product boundary and derive optimal compensation plans [8]. The proposed shrinkage model consists of two main sections known as systematic shrinkage that is considered constant and random shrinkage that can be predicted from experimented products using statistical approaches. To develop this model, first, Polar Coordinate System (PCS) is used to represent shape and shrinkage is defined as a parametric function denoting the difference between the nominal shape and the actual shape in different angles. Secondly, experiments are conducted and deformations are observed to derive the statistical distribution of the parameters in the function, based on which the shrinkage function can be defined and therefore compensation plans can be made. Figure 2 shows an illustration of the shrinkage model, a point $P$ on the nominal shape is represented in PCS as $r\left(, r_{0}, z\right)$, with $r$ and denoting its radius and angle, and $z$ denoting the z-coordinate of the $2 \mathrm{D}$ plane where PCS lies in. P', as the final position of $\mathrm{P}$ after shrinkage, can then be easily represented by reducing $r$ with a certain $r$, which is quite difficult to be identified under CCS. Compared with the work of Tong et al., this model reduces the complexity of deviation modeling by transforming the in-plane geometric deviations in CCS into a functional profile defined in PCS.

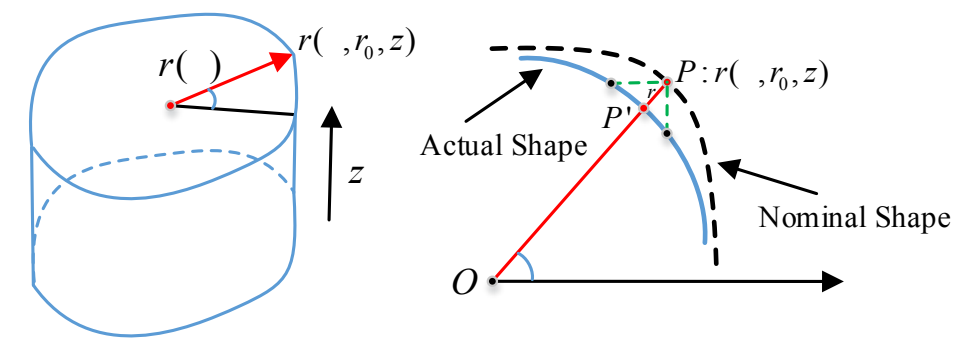

Fig. 2. In-plane shrinkage model in the Polar Coordinate System.

In a close study, Huang et al. [11] extends this approach from cylindrical shape to polyhedrons. They propose to treat an in-plane polyhedron as part of its circumscribed circle that can be carved out. A novel cookie-cutter function is proposed to be integrated in the cylindrical basis model to determine how the polyhedron can be trimmed from its circumscribed circle. This function is defined as a periodic waveform that can be modified according to the polyhedron shape. Later, this model is further extended to freeform shapes [12]. The freeform shape is approximated either by a polygon using the Polygon Approximation with Local Compensation (PALC) strategy or by addition of circular sectors using the Circular Approximation with Selective Cornering (CASC) strategy. Both of the strategies can 
be easily implemented based on previous models. Moreover, in [13] they propose a novel spatial deviation model under the Spherical Coordinate System (SCS), in which both in-plane and out-of-plane errors are incorporated in a consistent mathematical framework. Based on the above-mentioned shape deviation models, due compensation plans are made through experimentation using a stereolithography process. Overviewing all the publications of Huang and his co-authors, we can conclude that their research on the shape deviation modeling of AM process is generic and comprehensive, covering both 2D and 3D deviations, and shapes of different complexities. The methodologies have also been validated through extensive experiments, showing effective predictability of the shape shrinkage deviations. However, in order to deduce the exact functions of these models for compensation, measurements have to be made on certain number of experimental parts to obtain the shape deviation information. This means that these models can only be applicable when manufactured products are available. The models lack a consideration of the overall digital chain of the AM process and the ability to predict possible deviations without experimental data.

\subsection{Modification of input files}

Despite the error compensation approaches which seek to reduce geometric errors by learning from experimental data, a number of researchers have proposed to eliminate the errors in input files of AM processes. The modification of STL files and the improvement of slicing techniques are two mainstream research topics. These researches are motivated by the fact that AM does not work on the original CAD model, while uses the STL file in which the nominal part surface is approximated into a triangular mesh representation. A "chordal error" interpreted as the Euclidean distance between the STL facet and the CAD surface is introduced during the translation from CAD to STL, as can be seen from Figure 3(a). Besides, a "staircase error" occurs due to the slicing of the STL file in building the part layerby-layer, as shown in Figure 3(b). The maximum cusp height has been adopted as an accuracy measurement parameter for AM processes.

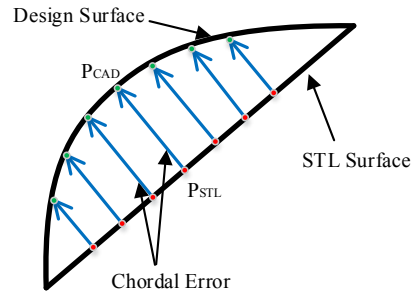

(a)

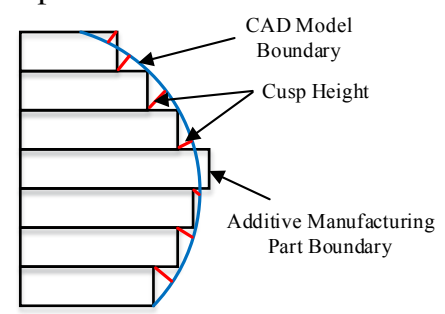

(b)

Fig. 3. (a) 2D illustration of the chordal error (b) 2D illustration of the staircase effect. 
A notable breakthrough in reducing chordal error is the Vertex Translation Algorithm (VTA) proposed by Navangul et al. [14], in which multiple points are selected on an STL facet and the chordal error is computed as the distance between each point and its corresponding point on the NURBS patch of the CAD surface. The point with the maximum chordal error is then identified and translated to the CAD surface, three new facets are generated by connecting the translated point with the vertices of the facet and then added to the STL file, while the original facet is deleted. Figure 4 gives an illustration of this algorithm. A facet isolation algorithm (FAI) is also introduced to determine the points to be modified by extracting the STL facets corresponding to the features of the part. This algorithm improves the STL file quality by iteratively modifying the STL facets until choral errors are minimized. However, numbers of iterations are usually required to satisfy the specified tolerance parameters and each iteration consumes significant amount of computation time and enlarges the file size. Similarly, the Surfacebased Modification Algorithm (SMA) proposed by Zha et al. [15] modifies the STL file by adaptively and locally increasing the facet density until the geometrical accuracy is satisfied. The individual part surfaces to be modified are selected by estimating the average chordal error and cusp height error of the surface. The modification is also applied on certain points of each STL facet of the selected surface according to predefined rules. However, SMA will likely increase the STL file size of a surface exponentially, so it is only preferable for high accuracy part models with complex features and multiple surfaces as a result of the tradeoff between smaller file size and higher accuracy.

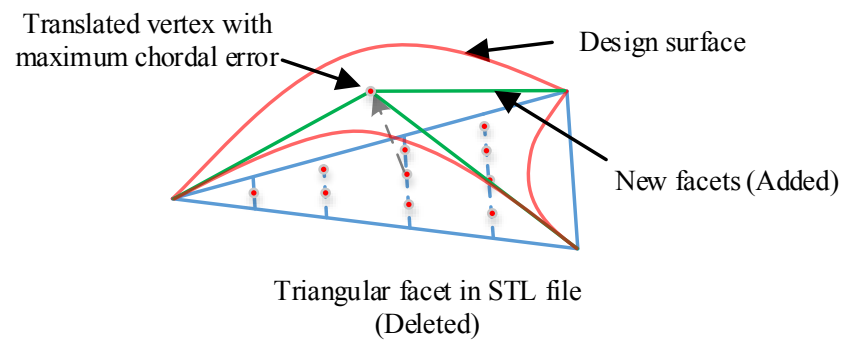

Fig. 4. Illustration of the VTA algorithm.

Instead of modifying the whole STL file, Kunal [16] proposes to minimize the errors by modifying each 2D slice of the STL file. The STL contour and designed NURBS contour on each slice are captured, and new points are generated on each triangle chord of the STL contour to verify if the chordal error threshold is exceeded in this chord. If so, corresponding chord points need to be translated to the NURBS contour until the chordal error is below the threshold. Thus new STL contour is formed by connecting the new points. This approach can be seen as a 2D version of the VTA algorithm that focuses on altering the part in manufacturing level. The modification is done on each slice, thus calling for large computation 
effort. Moreover, if any changes are made to the slicing plan, the whole process has to be repeated, which reduces its generality and constrains its application.

To reduce the staircase error, some researches on adaptive slicing have been conducted. Instead of uniform slicing which slices the STL file with a constant slice thickness, adaptive slicing seeks to slice the file using variable slice thicknesses to achieve the desired surface quality and at the same time ensure a decreased build time. The Octree data structures have been adopted by Siraskar et al. [17] to successively accomplish the adaptive slicing of the object. A method termed as Modified Boundary Octree Data Structure is used to convert the STL file of an object to an Octree data structure, by iteratively subdividing a universal cube enclosing the STL file into small node cubes according to the defined subdivision conditions. The height values of the final cubes can then be identified as slice thicknesses. This approach proves to have ensured the geometrical accuracy of the manufactured part through virtual manufacturing but is quite limited in real practice due to the lack of proper support for adaptive slicing in mainstream AM machines. To overcome this limitation, a clustered slice thickness approach is intuitively introduced [18], in which clustered strips of varying layer thicknesses are calculated manually using the minimum slice thickness, with each clustered band of uniform slices considered as a separate part built on top of each other along the build direction. A KDtree data structure is also adopted in this study to subdivide the bounding box of the STL file to determine the slice thickness. The cusp error threshold is used to decide whether the cube should be further subdivided. The adaptive slicing approaches are theoretically useful in reducing build time and increasing part accuracy, but the main challenge is the lack of direct support in AM machines.

\subsection{Evaluation and tolerance verification of shape deviations}

The deviations resulting from the proposed models need to be verified with respect to tolerance specifications, so as to provide significant feedback for the modification of design. Current studies focus on evaluating the deviations through analysis of the STL file. The STL file is sliced and the points on each slice contour are sampled for the evaluation. [19] proposes verification methods for both dimensional and geometric tolerances in AM processes. For dimensional tolerance, the Least Squares (LSQ) Fitting method is used to derive a substitute geometry of the extracted points, the dimension of which is then measured and verified. While for geometric tolerances, a Minimum Zone (MZ) Fitting method is used to derive the two separating nominal features which enclose all the extracted points with a minimum distance, and their distance is compared with the tolerance value. But this study overlooks the staircase effect and the MZ method is not realistic for shapes with complex geometries. In a series of work $[14,15,16]$, similar virtual manufacturing methods based on the STL file are adopted to evaluate shape deviations, in which the contour points in each slice is offset in the build direction for one-layer 
thickness to form a virtual layer, thus considering the staircase effect. The profile error of complex shapes is evaluated by calculating the maximum distance between contour points and their closest corresponding points on the design surface.

\subsection{Discussion}

In this section, two major categories of shape deviation modeling methods are reviewed and a comprehensive overview of these methods can be drawn as shown in Table 2. The validity of these methods has been proved through experiments and simulations. However, they could only cover certain phases of the AM process, while lacking the ability to model deviations from an overall view of the product lifecycle.

Table 2. Overview of the reviewed methods.

\begin{tabular}{llll}
\hline References & Dimensionality & Geometric Model & Main Characteristics \\
\hline$[4,5]$ & 2D, 3D & discrete & Machine errors of FDM and SLA \\
{$[6,7]$} & 2D & continuous & Modeling with PCS \\
{$[8-12]$} & 2D & continuous & Shape shrinkage, freeform shapes \\
{$[13]$} & 2D, 3D & continuous & Modeling with SCS \\
{$[14,15]$} & 3D & discrete & Modifying STL facets \\
{$[16]$} & 2D & discrete & Modifying 2D slice contours \\
{$[17,18]$} & 3D & discrete & Adaptive slicing \\
\hline
\end{tabular}

\section{The Skin Model Shapes paradigm for AM}

The main contributions of the Skin Model Shapes have been highlighted recently in different applications, such as assembly, tolerance analysis, and motion tolerancing. The generation of Skin Model Shapes can be divided in a prediction and an observation stage depending on the available information in product design and manufacturing processes [2]. Geometric deviations can be classified into systematic and random deviations, where systematic deviations originate from characteristic errors of the manufacturing process, while random deviations occur due to inevitable fluctuations of material and environmental conditions. Notably, in the observation stage, geometric deviations are modeled by extracting the statistical information of deviations from a training set of observations gathered from manufacturing process simulations or measurements, thus possible deviations in newly manufactured parts can be effectively predicted [20]. Figure 5 shows the creation of Skin Model Shapes in both prediction and observation stages. 


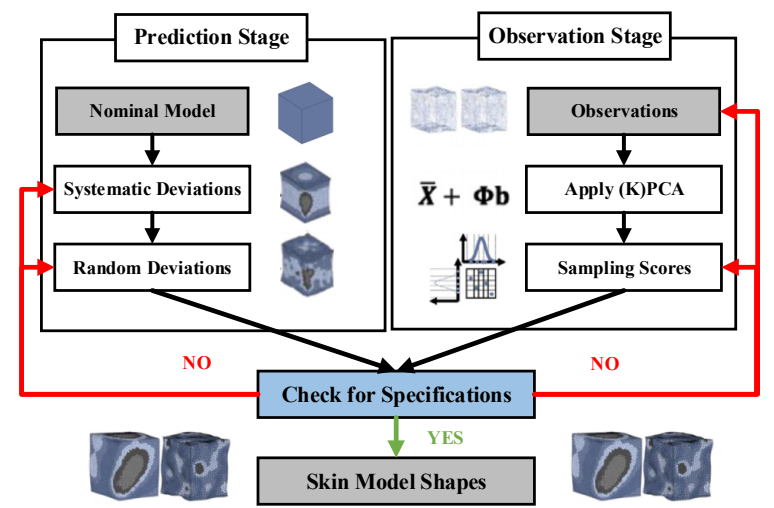

Fig. 5. The creation of Skin Model Shapes in prediction and observation stages[19].

Compared with the above mentioned approaches, the Skin Model Shapes paradigm can model both the 2D and 3D deviations either by prediction based on assumptions and virtual or real experiments, or by learning from observation data gathered from manufactured samples. It covers the overall digital chain of the AM process, though specific methodologies for its application in AM remain to be developed, it is a suitable and promising modeling framework for AM processes.

\section{Conclusion}

Geometrical accuracy is an important concern for AM processes. In this paper, current research status of shape deviation modeling of AM processes is reviewed. Major modeling methods, categorized as Deviation Compensation methods and Input File Modification methods, have been discussed and their advantages and limitations are highlighted. The Skin Model Shapes paradigm is introduced to be a promising modeling method for AM. In further studies, authors will focus on adapting the Skin Model Shapes paradigm to the AM process and come up with a comprehensive deviation modeling framework to support the whole digital chain.

Acknowledgments This research has benefitted from the financial support of China Scholarship Council (first author).

\section{References}

1. ASTM F2792-12a, Standard Terminology for Additive Manufacturing Technologies, ASTM International, West Conshohocken, PA, 2012.

2. Anwer N., Ballu A., and Mathieu L. The skin model, a comprehensive geometric model for engineering design. CIRP Annals-Manufacturing Technology, 2013, 62(1), 143-146. 
3. Garaizar, O. R., Qiao, L., Anwer, N., and Mathieu, L. Integration of Thermal Effects into Tolerancing Using Skin Model Shapes. Procedia CIRP, 2016, 43, 196-201.

4. Tong K., Amine Lehtihet E., and Joshi S. Parametric error modeling and software error compensation for rapid prototyping. Rapid Prototyping Journal, 2003, 9(5), 301-313.

5. Tong K., Joshi S., and Amine Lehtihet E. Error compensation for fused deposition modeling (FDM) machine by correcting slice files. Rapid Prototyping Journal, 2008, 14(1), 4-14.

6. $\mathrm{Xu}$ L., Huang Q., Sabbaghi A., and Dasgupta T. Shape deviation modeling for dimensional quality control in additive manufacturing. In ASME 2013 International Mechanical Engineering Congress and Exposition, IMECE 2013, San Diego, November 2013, pp. V02AT02A018-V02AT02A018.

7. Song S., Wang A., Huang Q. and Tsung F. Shape deviation modeling for fused deposition modeling processes. In IEEE International Conference on Automation Science and Engineering, CASE 2014, Taipei, August 2014, pp.758-763.

8. Huang Q., Zhang J., Sabbaghi A., and Dasgupta T. Optimal offline compensation of shape shrinkage for three-dimensional printing processes. IIE Transactions, 2015, 47(5),431-441.

9. Huang Q., Nouri H., Xu K., Chen Y., Sosina S., and Dasgupta T. Statistical Predictive Modeling and Compensation of Geometric Deviations of Three-Dimensional Printed Products. Journal of Manufacturing Science and Engineering, 2014, 136(6), 061008.

10. Sabbaghi A., Dasgupta T., Huang Q., and Zhang J. Inference for deformation and interference in 3D printing. The Annals of Applied Statistics, 2014, 8(3), 1395-1415.

11. Huang Q., Nouri H., Xu K., Chen Y., Sosina S., and Dasgupta T. Predictive modeling of geometric deviations of $3 \mathrm{~d}$ printed products-a unified modeling approach for cylindrical and polygon shapes. In IEEE International Conference on Automation Science and Engineering, CASE 2014, Taipei, August 2014, pp.25-30.

12. Luan H. and Huang Q. Predictive modeling of in-plane geometric deviation for 3D printed freeform products. In IEEE International Conference on Automation Science and Engineering, CASE 2015, Gothenberg, August 2015, pp.912-917.

13. Jin Y., Qin S.J., and Huang Q. Out-of-plane geometric error prediction for additive manufacturing. In IEEE International Conference on Automation Science and Engineering, CASE 2015, Gothenberg, August 2015, pp. 918-923.

14. Navangul G., Paul R., and Anand S. Error minimization in layered manufacturing parts by stereolithography file modification using a vertex translation algorithm. Journal of Manufacturing Science and Engineering, 2013, 135(3), 031006.

15. Zha W. and Anand S. Geometric approaches to input file modification for part quality improvement in additive manufacturing. Journal of Manufacturing Processes, 2015, 20, 465477.

16. Sharma K. Slice Contour Modification in Additive Manufacturing for Minimizing Part Errors. Electronic Thesis or Dissertation. University of Cincinnati, 2014. OhioLINK Electronic Theses and Dissertations Center. 03 April 2016.

17. Siraskar N., Paul R., and Anand S. Adaptive Slicing in Additive Manufacturing Process Using a Modified Boundary Octree Data Structure. Journal of Manufacturing Science and Engineering, 2015, 137(1), 011007.

18. Panhalkar N., Paul R., and Anand S. Increasing Part Accuracy in Additive Manufacturing Processes Using a kd Tree Based Clustered Adaptive Layering. Journal of Manufacturing Science and Engineering, 2014, 136(6), 061017.

19. Moroni G., Syam W.P., and Petrò S. Towards early estimation of part accuracy in additive manufacturing. Procedia CIRP, 2014, 21, 300-305.

20. Anwer N., Schleich B., Mathieu L., and Wartzack, S. From solid modelling to skin model shapes: Shifting paradigms in computer-aided tolerancing. CIRP Annals-Manufacturing Technology, 2014, 63(1), 137-140. 